\title{
Songs of Dissent and Consciousness: Pronouncements of the Bauls of Rural Bengal
}

\author{
Uttaran Dutta ${ }^{1, * \mathbb{D}}$, Panchali Banerjee ${ }^{2}$, Soham Ghosh ${ }^{3}$, Priyam Ghosal ${ }^{3}$, Samya Srimany ${ }^{3}$ and \\ Sahana Mukherjee ${ }^{3}$
}

check for updates

Citation: Dutta, Uttaran, Panchali Banerjee, Soham Ghosh, Priyam Ghosal, Samya Srimany, and Sahana Mukherjee. 2021. Songs of Dissent and Consciousness: Pronouncements of the Bauls of Rural Bengal. Religions 12: 1018. https://doi.org/ 10.3390/rel12111018

Academic Editor:

Heather MacLachlan

Received: 31 July 2021

Accepted: 14 November 2021

Published: 18 November 2021

Publisher's Note: MDPI stays neutral with regard to jurisdictional claims in published maps and institutional affiliations.

Copyright: (C) 2021 by the authors. Licensee MDPI, Basel, Switzerland. This article is an open access article distributed under the terms and conditions of the Creative Commons Attribution (CC BY) license (https:// creativecommons.org/licenses/by/ $4.0 /)$.
1 Hugh Downs School of Human Communication, Arizona State University, Tempe, AZ 85287, USA

Department of Economics, Jadavpur University, Kolkata 700032, India; panchali.banerjee89@gmail.com Department of English, Jadavpur University, Kolkata 700032, India; tyrionsoham@gmail.com (S.G.); priyamghosal@gmail.com (P.G.); samya2624@gmail.com (S.S.); sahanamukherjee95@gmail.com (S.M.)

* Correspondence: uttaran.dutta@asu.edu

\begin{abstract}
Bauls, the wandering minstrels of rural Bengal (of both Bangladesh and India), are a socioreligiously marginalized cultural group. While the ritualistic practices and spiritual discourses of the Bauls have received scholarly attention, scholarship on Bauls' songs about material and communicative adversities and their emancipatory visions is lacking. Bauls' performances and discourses are precursors to envisioning alternative emancipatory possibilities that question dominant intolerances, oppressions, and exploitations. This article documents and reflects on the works of two contemporary Bauls-Shah Abdul Karim and Manimohan Das. Through their songs and performances, they (i) question the power structure and legitimize the sufferings and struggles of the downtrodden, and (ii) seek to raise societal consciousness in imagining a free and just society.
\end{abstract}

Keywords: Baul; voice; Bengal; emancipation

\section{Introduction}

\section{তত্ত্বগান গেয়ে গেলেন যারা মরমী কবি \\ আমি তুলে ধরি দেশের দুঃখ-দুর্দশার ছবি নিরন্ন মানুষের দাবি- করিম চায় শান্তি বিধান।}

The contemplative poets are they who sang the songs of tattwas, In my songs, I portray images of people's misery and grief,

And, the demands of the famished,

Karim wants a peaceful co-existence

Tattwas are spiritual theories and epistemologies - Baul Shah Abdul Karim

Bauls, the wandering minstrels, are one of the socio-religiously marginalized cultural populations of rural Bengal, from both Bangladesh and the eastern/north-eastern regions of India (Salomon 1995). The poets, performers, and followers that belong to Hindu and Muslim societies are called Bauls and Fakirs, respectively; but in general, the term Baul is used to represent Bauls, Fakirs, and practitioners of similar spiritual traditions. On 25 November 2005, UNESCO added Baul songs to the global list of 'Masterpieces of the Oral and Intangible Heritage of Humanity' (Khalid and Chowdhury 2018).

Historically, dominant oppressions (including colonial exploitations) and castepolitics are responsible for socio-cultural and politico-economic uncertainties in the lives of Bauls. Scholars have shown that organized religions, on many occasions, confronted and abused the followers of non-traditional and folk religions. One can even find such examples from 5th and 6th century BCE, such as in the experiences of Charvakas and Ajivikas (Mondal 2013). Be they followers of Kabir, Dadu, Rabidas, Nanak, Chaitanya (religious 
teachers from 14th/15th Century) or contemporary Bauls, the alternative religious practitioners express dissent against religious dogma and discrimination, especially related to the caste system and the Hindu-Muslim divide, while embracing the ideas of equality, freedom, and harmonious coexistence (Lorea 2014). In response, intolerant orthodoxies of organized religions, through their physical and discursive violence, have aimed to mute and even obliterate the free thought and emancipatory dreams of alternative spiritual practitioners, such as the Bauls. The murder of Mohammed Shahidulla in 2016 and the torching of Baul Ranesh Thakur's house (a direct disciple of Baul Shah Abdul Karim) in 2020 are examples of such atrocities (Dutta 2019; Shaker 2020). In other words, the Bauls of Bengal are systematically harassed and stigmatized, and as outcasts, they remain at the margins of South Asian society (Akter et al. 2017).

While we have noticed increased scholarly attention to the ritualistic practices and epistemologies of the Bauls, the existing scholarship on Bauls' songs about material and communicative adversities and emancipatory visions are scant (Dutta 2019). Moreover, the bulk of the extant scholarship has cursorily engaged with the socio-economic and political marginalization of the rural Baul communities, most of whom live in poverty. It is important to recognize the few yet powerful non-esoteric Bauls songs, which, along with depicting the everyday struggles of the marginalized, play a crucial role in raising critical consciousness and imagining human transformation. Creators of such Baul songs venture beyond the realm of the tattwa represent humane conscience and narrate the adversities of helpless people at the margins (Mondal 2013). On one hand, these songs communicatively fight against social exclusions and atrocities to question dominant intolerances and injustices. On the other hand, such songs and performances raise consciousness in imagining alternate emancipatory possibilities, as well as in creating a less exploitative and a more egalitarian society (Urban 2001).

The contribution of this article lies in its documentation and reflection on the songs and discourses of two contemporary Bauls-Shah Abdul Karim and Manimohan Das. Shah Abdul Karim was a legendary Baul poet and performer from Bangladesh who embraced the Baul/Fakir philosophy. He embodied the virtues of simplicity, modesty, honesty, and humanity through his songs and portrayed experiential realities and disparities of everyday existence at the margins. Likewise, Manimohan Das, a Baul from West Bengal in India, wrote songs that talked about the social injustices of the contemporary era, along with their nuances and complexities. Being cognizant about the history and trauma of social exclusions, and oppressions, Baul Das and Karim, on one hand, through their songs and performances, spoke to the socio-economic issues at the margins by questioning the dominant power structure. On the other hand, they legitimized the suffering and struggles of the downtrodden and raised societal consciousness to imagine a free and just society. It is these pronouncements that constitute the focus of this paper.

Specifically, embracing the theoretical perspectives of Subaltern Studies, especially Gramscian viewpoints, Scott's 'hidden transcripts', and Freirean perspectives, this paper examines the songs of the two contemporary Bauls to examine how their pronouncements (i) illustrate situated adversities and disparities, as well as the negotiations of the underserved people with societal challenges, and (ii) communicate a message of emancipation and meaningful transformation to envision and realize the dreams of an exploitation- and oppression-free future. In doing so, we focus on analyzing the literary works of the Bauls, specifically the song-lyrics, and support our analysis of the lyrics with statements from interviews with Bauls Das and Karim (rather than espousing an ethnographic or performance studies perspective).

\section{Literature Review}

Scholars have noted that Sants (Bhakti movements), Naths, Buddhists, Jains, Besra Sufis, and several minor sects of the Indian sub-continent are predecessors of the contemporary Baul tradition (Lorea 2015). Charyāpada (Buddhist esoteric songs from 8th-12th century), medieval Sant poetry, or dohās, Nath literature, as well as the literary works of 
Chaitanya Charitāmrita and Sahajiya Karicā can be seen as foundational to the literary tradition of the Bauls (Capwell 1974; Lorea 2014). Many of these texts addressed experiential learnings, rather than blindly following "doctrinal or dogmatic niceties" (Capwell 1988, p. 124), and they placed emphasis on spiritual catharsis. They imagined an equal society devoid of caste-related and other discriminations and oppressions by arguing in favor of human rights (Urban 2001). Owing to such anti-hegemonic and uncompromising tones, the orthodox Hindu and other major religious traditions usually term their practices as Apadharma or Apasampraday (an inferior/impure religion or religious group). The dominant groups often register their hatred and rejection by attacking them systematically, as well as strategically, to obliterate such thought-traditions and ideologies (Capwell 1974; Dutta and Dutta 2019). In addition, various puritanical efforts by powerful religions (e.g., Sanskritization and Islamic fundamentalism) seek to co-opt alternative and folk religious traditions, including those of the Bauls, bringing them into the fold of the mainstream. In response, similarly to their Lokayata predecessors, many such practitioners hide and/or camouflage to save and/or sustain their spiritual practices and ideologies (Mondal 2013).

Consequently, over the centuries, impacted by the politics of exploitation and exclusion by the upper-castes, dominant religions, and colonial rulers, the lower socio-economic strata of the society experienced laborious uncertainties and sufferings. In response, many "deviant sects", including the Bauls, emerged in various parts of Bengal (Urban 2001). Historically, the Bauls have experienced fatwas and threats from the mainstream, which intend to abuse, co-opt, and banish their non-conformist practices (Manuel 2008; Jha 2014). As they do not comply with the normative traditional canons of established religions, the Bauls and their songs are often depicted as heretic, cultish, rebellious, and as infidels. As a result, the Bauls have been harassed and stigmatized over the years (Grover 2015; Novetzke and Patton 2008).

While coping with atrocities and exclusions, the Baul community has received a degree of acceptance among the educated, urban Bhadralok or Bengali gentlemen, after their philosophies and worldviews were admired in literary works of Tagore, a Nobel Prize winner in literature, and Nazrul Islam, the national poet of Bangladesh, and other important cultural personalities in Bengal. From Tagorean perspectives, the Bauls are perceived as an icon of an inclusive and secular tradition, where the Quran and Puran can peacefully co-exist, acting as an embodiment of simplicity, tranquility, and equality, which exemplified an ideal nationalist identity during the period of the Indian independence movement (Lorea 2013). In contemporary literature, the Bauls are often described as lower-class, under-privileged and uneducated in terms of formal education, yet as peace-loving and non-conformists. Moreover, in the last few years, we have noticed a resurrection of religious orthodoxies and fanaticism in the South Asian landscape which has caused distress and misery at the margins. By rupturing the barriers of caste and religion, the songs of the Bauls calling for love and peace are re-emerging as relevant at this turbulent time. In this technology-savvy, globalized era, we have seen a proliferation of fake-bauls/urbanbauls (educated middle-class individuals who consider singing as a side job), particularly in socially mediated spaces. Owing to these newly emerging dynamics, many marginalized and traditional Baul practitioners experience difficulties in ensuring their material and spiritual survival (Lorea 2014).

In negotiating their low-origins, and the oppression and humiliation they are subject to, the Bauls consider songs and spirituality as their last refuge (Urban 2001). Despite being numerically small and socio-economically disadvantaged as a community, the Bauls assume a duty to consistently convey their philosophical and behavioral teachings to the masses (Mondal 2013). Horizontal and participatory communication is a central element of their praxis. In the same way as the Siddhacharyas - the writers of Charyāpadas who wrote in the regional vernacular rather than in the dominant/canonical language - the Bauls and Fakirs also perform in local languages to orally communicate with the common people (Majumdar and Roberge 1979). Their simple and rustic music fulfills important social functions, as the Bauls often seek to communicate and create social awareness about 
everyday matters. The inner and layered meanings of their songs, specifically those that are non-esoteric, aim at propagating the values of harmonious coexistence, social justice, and environmental consciousness (Mahbub-ul-Alam et al. 2014). The Bauls' renderings of alternative spiritualities and their questioning/skepticism despite harsh opposition and attacks from the hegemonic powers can be examined through the lens of subaltern studies and related theories.

According to the Gramscian viewpoint, which is foundational to the theories of Subaltern Studies, alternative spiritual practices and principles are not mere 'sediments' of mainstream intellectual and religious traditions. Rather, they emerge organically and experientially through conscious observation and active interaction with contextual realities (Patnaik 2014). Rooted in Gramscian perspectives, the cultural dimensions of subalternity were initially under-emphasized in economic-centric conceptualizations of Indian Marxism (Chakraborty 2000). Consequently, in much of the former canon of subaltern studies, aspects of subaltern spirituality are often ignored or have received inadequate attention (Urban 2001). Today, the scholars of subaltern studies acknowledge that "religious consciousness is a vital subject of study" (Larson 1995, p. 41). Situated cultural (including religious) practices and ethos have now become foundational to subaltern study scholarship, so as to legitimize the narratives and agencies of those existing at the margins, as well as to foreground their de-colonial struggle for socio-cultural and political liberation (Wang 2010).

The Bauls' cultural, specifically musical, expressions and ideological currents can be considered as one such example, distinctively different from those of mainstream religious organizations and their praxis. Gramscian theorists further posited that situated religiocultural utterances can often constitute a tradition of protest, the roots of which can be traced to a variety of locations and spaces, including those that are unobvious and contradictory, associated with subaltern experiences (Patnaik 2014). From a Gramscian lens, these experiences are intertwined with the religious common sense of subaltern populations, whose everyday consciousness is often expressed through their performances, questionings, rituals, customs and mythologies (Fulton 1987). Therefore, according to Patnaik (2014), it is crucial to recognize the various unorthodox loci of counter-hegemony, which are instrumental in imagining alternative realities, as well as paving emancipatory and liberating avenues. He further envisioned Bauls and practitioners of similar traditions as "'organic intellectuals' from subaltern strata" (p. 22) and noted that their perspectives and creativities are foundational to create counter-hegemonic possibilities in an autonomous way.

In addition, it is important to understand religio-cultural performances and emancipatory imaginings of underrepresented spiritual communities, such as Bauls, not as a vehicle of openly pronounced and violent revolution, but as a subtler, passive, and less conspicuous expression of resistance (Kumar 2019). In this context, Scott's (1990) work on passive, everyday resistance is pertinent in understanding the approaches and the actions of marginalized populations, such as the Bauls. He noted that an indirect and less violent form of resistance, which he termed as 'hidden transcripts', often "takes place 'offstage', beyond direct observation by the powerholders" (p. 4). Embracing Scott's perspective, scholars have noted that the Bauls use their songs and compositions as a means of resistance, through which they question and challenge existing disparities, intolerance, and inequalities discursively, without using any radical form of resistance, such as a peasant revolt (Mukharji 2012). Scott (1990) further showed that such resistive acts can be communicated by underprivileged groups in a variety of forms, including through songs and performances.

In their praxis, the Bauls do not reject the mainstream bluntly or propagate negativity; rather, guided by their ideologies, commitments, and practices of seeing and acting, they often strategically operate, primarily communicatively, behind the guise of mainstream practices. Although the resistances of the Bauls are often of a small scale, less visible, cryptic, and even private, these strategic acts potentially help them to spiritually sustain, as 
well as communicate musically with the audience (Mukharji 2012). In other words, the Bauls use their quotidian, non-violent, and certainly not insignificant forms of resistance to address various structural and material, as well as cultural and intangible, aspects of contextual realities. By discursively referring to socio-religious symbols, they often question the dominant power's construction of otherness and subalternity (Mukharji 2012). Embracing the dreams of an egalitarian, free, and just social order, the Bauls (especially, the singers of non-esoteric songs) interact closely with the masses on a regular basis to raise voices and question the existing sociopolitical dynamics and the status quo.

To engage with the Bauls' politics of emancipation, it is crucial to understand the aspect of consciousness raising, as theorized by Paulo Freire (1970). According to Freire, critical consciousness acts as a means by which to challenge and dismantle the culture of silence in order to engender transformative possibilities. Freire (1970) showed that, by reclaiming the culture of thinking and responding, the conscientization processes can help to develop and reinforce self-awareness. In the context of the Bauls, songs can be seen as discursive vehicles that create a moment of dialogue. These songs eventually help these subaltern performers to co-create possibilities to enhance solidarity, communion, and building social capital to pave the ways for cooperation to the end of developing human values and liberatory mindsets. That is, through understanding the meanings, causes, ideologies, and consequences of actions, the alternative spiritual imaginings seek to raise consciousness of the underserved populations to address contextual realities and adversities.

In the case of the Bauls, the images, illustrations, and metaphors used in their songs are oftentimes examples of 'hidden transcripts' that typify covert forms of everyday resistance. Moreover, as a part of their practices and behavior, quotidian public interactions can be seen not only as their response to endless subjugation, but also as their relentless struggle toward imagining an equal and peaceful society, where human freedom and emancipation are celebrated.

\section{Bauls}

The confluence of three spiritual traditions - Tantric Buddhism (Vajrayana and Sahajyana), Hindu Vaishnavism, and Islamic Marifat, marks the foundational epistemology of the Bauls (Lorea 2017). Guided by the aforementioned traditions, in praxis, the Bauls often question/reject discourses of orthodox religions (Openshaw 2002). For instance, Bauls' ideology does not value the ritualistic worship of deities or symbols, and/or give importance to religious spaces, such as mosques or temples. Moreover, through their songs, they strongly criticize the 'caste system' and other forms of social injustice (Sengupta 2015). In response, they often experience severe threats from both the dominant Hindu and Islamic authorities; one of which is a 'Baul Dhangsher Fatwa', a fatwa for demolishing Baul tradition (Jha 2014).

Although they are mostly unlettered, the Bauls exhibit distinctive aesthetical and performative talents (Hossain and Marinova 2005). Along with communicating socioreligious and spiritual discourses, the Bauls, and specifically the composers and performers of non-esoteric songs, also raise their voices to address contemporary topics, including poverty, hunger, corruption, domestic abuses, health and hygiene and deforestation. Such songs are composed not to simply entertain the audience, rather, the performers invite common people to embrace the path of reflexivity and to remain close to nature in their endless struggle against coercion and manipulation (Mondal 2013). Through poetic and musical discourses, they voice aspects of cultural poverty, specifically in terms of ethics, values, and spirituality, and call for introspective engagements in everyday existence. As a collective of a heterogeneous and non-institutional community of singers and practitioners, these Bauls gently yet persuasively convey their counterculture discourses, oftentimes against capitalistic values, mindless industrialization, and urbanization (Majumdar and Roberge 1979). As previously mentioned, this paper presents the songs and discourses of two contemporary Bauls-Shah Abdul Karim and Manimohan Das, who composed and performed during 20th and 21st centuries, and raised their voices against existing forms 
of domination and exploitation, to communicate their dreams of an emancipated and liberated society.

\subsection{Baul Manimohan Das}

Born in Faridpur (present day Bangladesh) in 1956, Baul Manimohan Das travelled to India when he was 6 years old. During his childhood, he experienced extreme economic hardships and hunger; to stay alive, he worked as a domestic worker. He did not have access to any formal modes of education. He overcame the barrier of illiteracy by himself. By imitating written texts, he learned to read and write. As he grew older, he developed a habit of reading books, writing poems/songs, and performing in public. Religious texts, such as the Bhagavat Puranas and Gita, and Baul-praxis influenced him to a great degree. As an adult, he used several means to ensure survival and stayed at various locations across South Bengal. While working hard to overcome adversities, he has consistently mingled with Baul practitioners and written relentlessly. Thus far, he has written more than 4000 songs. 'Gramin Sangiter Dali' (Das 2014) is his most prominent publication, which contains around one-fifth of his writings.

\subsection{Baul Shah Abdul Karim}

Born in 1916 in the Sunamganj district of Bangladesh, Baul Shah Abdul Karim was a venerable Baul singer and spiritual composer. Growing up living in poverty and resourcescarcity, he never had an opportunity to pursue a formal education in school. To survive amidst harsh realities and adversities, he worked as a cow herder in the houses of local affluent people. While struggling with abject poverty, he embraced humane values and Baul ideologies early in his life. He began expressing his experiences and realizations through music at a young age and continued his composing and singing activities throughout his life. He wrote thousands of songs, many of which were organized into six books, while numerous undocumented songs have been lost. He was an active advocate of human rights, social justice, and liberation, and he consistently conversed and interacted with common people. His songs and words captivated audiences and inspired people to imagine and create avenues for emancipation. For his unique contributions, he was awarded 'Ekushe Padak', the second-highest civilian award in Bangladesh. He died in 2009 at the age of 93.

As they unconventionally focused on socio-political injustices, disparities, and abuses, and sang about the struggles of the working class and the downtrodden, both men faced criticism during their lifetime; people from within and outside of their communities questioned their legitimacy as authentic Bauls. However, by referring to Lalon's acts of supporting self-defense and singing songs against social ills, some scholars supported their songs, approaches, and intentions, and portrayed them (specifically, Baul Shah Abdul Karim) as successors of eminent Bauls, such as Rashid Uddin, Jalal Khan, Dwija Das, and Ramesh Shil (Imam 2013). While it is difficult to use quantifiable indicators to measure tangible changes realized by the Bauls' endeavor for a free and just society, their struggle to create such realities have not been in vain or diminished. Along with performing songs of emancipation, both formed social organizations to ensure survival at and of the margins. Baul Shah Abdul Karim once shared, "With my limited ability, I run an organization in our area, named 'Banchte Chai' (Want to live). Our expectation is very modest; we just want to eat twice for survival. Unfortunately, availability of essential commodities such as food-grains is scarce; they are often controlled and snatched by the oppressors" (Imam 2013). Baul Manimohan Das founded an organization called 'Amrita Baul Lokgaan Prasar Samity', described, in his own words, "as an organization, we tried to address the issues of malnutrition, marginalization as well as create health and environmental awareness. In my life, I always tried, with my finite capacities, to help fellow artists and the marginalized people in and around my locality". 


\section{Methods}

We conducted an extensive literature search to identify Baul and Fakir related resources. For this purpose, primarily textual resources and some audio-visual files were examined; these resources were found via libraries and internet searches. After a comprehensive search, over 4000 Baul songs were located; we mainly relied on published books, including the song-collections of eminent scholars, such as Prof.s Upendranath Bhattacharya and Shaktinath Jha. The songs were grouped into two main categoriesdevotional, or socio-political with emancipatory connotations. As a part of the search process, we identified two resources written by two contemporaries, Baul Shah Abdul Karim and Baul Manimohan Das, who composed and performed in the 21st century. Those two books contained over 1200 songs, many of which represented the issues, aspirations, and dreams of the marginalized. We also included some interviews and conversations with the two Bauls, where they communicated their transformative thoughts and commitments. For this study, we considered only the discourses, including songs, which were about human suffering, social injustice, discrimination, as well as represented emancipatory visions. After careful reading, initially 40 songs and interviews were identified for content analysis for this paper.

From the historical record, we found that the songs were performed at public gatherings, and they were well received by the audience. In one interview, Baul Shah Abdul Karim remarked that, "during the struggle for freedom (Muktijuddho), I used to get invitations to perform at many gatherings and processions". Though they had been appreciated by people during that period, the songs are no longer not counted among those of the 'popular numbers', and we could not locate videos/audios of the songs analyzed in the article as they are unavailable through YouTube and other online platforms. Again, many of Shah Abdul Karim's songs were either lost or not published, so despite our efforts, we could not find his early published books. However, two aforementioned books (by Baul Karim and Baul Das) provided us the opportunity to read and research their less-known songs. As mentioned previously, many of their songs spoke to the issues of the era in which the songs were written. While identifying songs for this research, we selected those that capture and address the contemporary crisis and challenges in a more meaningful way than the others are able to. Moreover, in terms of their lyrics, unlike the tattwa-gaan(s), the songs are straightforward and non-esoteric in nature, that is, twilight language ${ }^{2}$ and layered meanings are never used.

As mentioned previously, this research is invested in studying and analyzing the lyrics and discourses. As the songs were written and interviews were conducted in the Bengali language and its dialects, we translated and transcribed them into English for the purpose of data analysis. After translations and transcriptions, we reviewed one another's work and provided feedback. Local proverbs and colloquial expressions were found in many of the songs and interviews. As we often faced difficulties in translating them, we consulted local Bauls (including Baul Manimohan Das) and scholars to ensure authenticity. The accuracy of the translation was examined by another scholar who was an experienced Bengali-to-English translator (Lincoln and Guba 1985). Any disagreements were resolved through discussions, and based on consensus, necessary modifications were made.

The data were analyzed by employing an approach based on grounded theory (Charmaz 2000). The discourses (i.e., the songs and the interviews) were analyzed closely; a constant comparison technique helped us to compare and contrast the emerging points and concepts. After close reading and careful analysis, 10 songs were identified for which the messages and contents did not overlap so that each selected song offered a unique vantage point. As the study is primarily invested in the discourses of songs, we present full transcripts of the songs in the Results section. The processes of data analysis yielded three themes: oppressions and marginalization, communicative aspects, and emancipatory visions and calls, which are presented in the next section. 


\section{Results}

The discourses of Bauls Karim and Das are deeply tied to their socio-historic experiences, particularly in relation to the dominant power structures both in the colonial and postcolonial era. In other words, their songs are the embodiment of fearless communication and autonomous consciousness, which exemplify acts of questioning, and resisting hegemonic agendas and oppressions. Praxiologically, both Bauls embrace the means of aesthetic expressions, where they employ communicative avenues to transform power dynamics. By dismantling the culture of silence, which delegitimizes human agencies and voices, their songs often reflect the values, worldviews, and aspirations of the common people, especially those who are marginalized.

\subsection{Oppressions and Marginalization}

To both Bauls, the ever-increasing gaps between the haves and the have-nots are deemed as unacceptable. More than two-thirds of the rural Bengal population lives below the poverty line (World Bank 2017). Moreover, owing to its geographical location and characteristics, a major proportion of Bengal (i.e., the state of West Bengal in India, and the entire Bangladesh) frequently experience natural calamities, such as cyclones, droughts, and floods. Such natural disasters impact the lives and livelihoods of millions of common people. Moreover, crop failure, soil erosion and loss of land, and financial instabilities make their situation worse (Hossain and Marinova 2012). In their songs, they portray a bleak reality and also communicate their criticism about the roles and (in)actions of the power structure. For instance, Baul Manimohan Das once said, "Since childhood, I experienced hunger and economic hardships. These experiences compelled me to raise a voice against the oppressions in this society". He further added, "I was concerned about societal inequalities, discriminations, lack of human rights, and abuse of law by the powerful. I started responding to the issues around me through my own songs by using simple and accessible language. I was deeply inspired by the principles of equality and social justice; these ideals guided me to write".

Alluding to feudalistic, capitalistic, and neoliberal practices, the following song composed by Baul Shah Abdul Karim speaks of heightening poverty in the face of ever-rising crises, with which the farmers and laborers must contend. The corrupt social structure applies different methods of oppression, wherein the poorer populations are viewed merely as electorates by nation-states. For instance, the promises made prior to elections are broken, and the poor populations are often controlled by the age-old practices of divide and rule.

Referring to food insecurities and frequent natural calamities in Bengal, the song legitimizes the dire situation of poverty-stricken people. The social services that are supposed to be publicly accessible are unfortunately more amenable to the wealthy than to the poor. Primary schools in rural areas are devoid of supplies and teachers, health centers have inadequate medicines, and so on. Baul Karim also argues that the government is invested in collecting taxes but not diligent in providing basic minimum necessities for survival. Instead, coercion and repression are practiced by the dominant stakeholders to mute marginalized people. Moreover, unsatisfactory and biased judgment is delivered to the poor because of their lack of resources or financial power. In this song, by using simple language, Baul Karim not only raises a voice against the situated odds, but also publicly communicates important ethical and moral issues. 


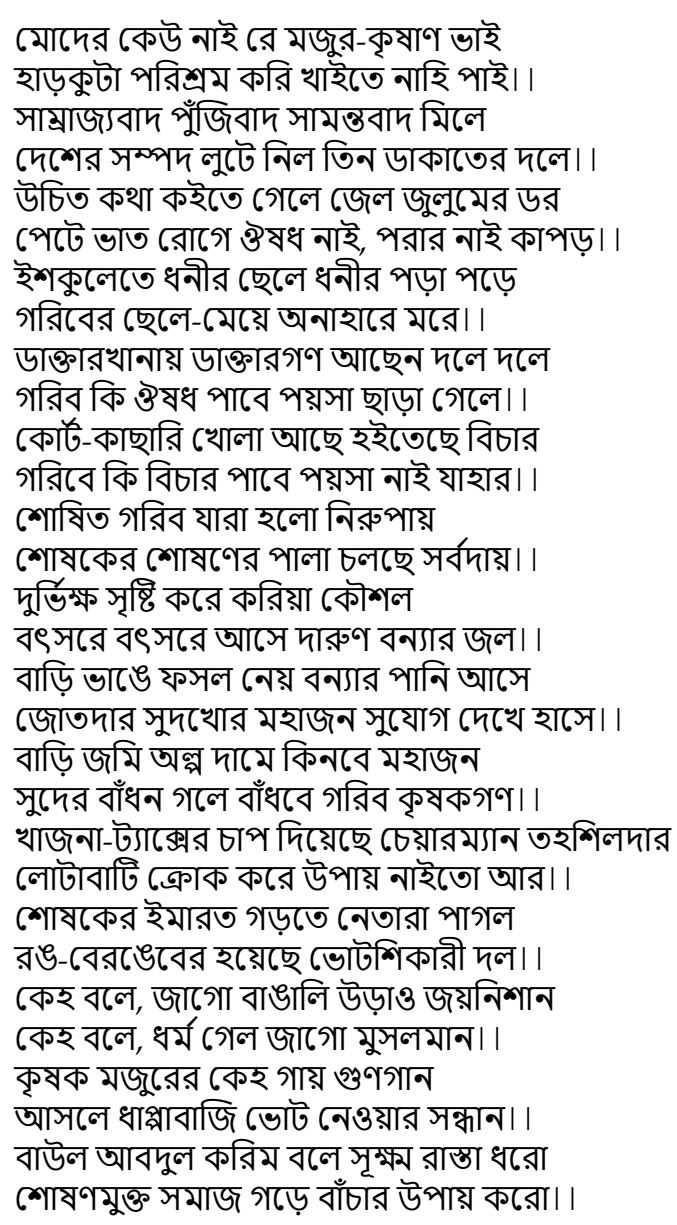

মোদের কেউ নাই রে মজুর-কৃষাণ ভাই হাড়কুটা পরিশ্রম করি খাইতে নাহি পাই।। সাম্রাজ্যবাদ পুঁজিবাদ সামন্তবাদ মিলে দেশের সম্পদ লুটে নিল তিন ডাকাতের দলে। উচিত কথা কইতে গেলে জেল জুলুমের ডর পেটে ভাত রোগে ঔষধ নাই, পরার নাই কাপড়। ইশকুলেতে ধনীর ছেলে ধনীর পড়া পড়ে গরিবের ছেলে-মেয়ে অনাহারে মরে।। ডাক্তারখানায় ডাক্তারগণ আছেন দলে দলে গরিব কি ঔষধ পাবে পয়সা ছাড়া গেলে। শোষিত গরিব যারা হলো নিরুপায় শোষকের শোষণের পালা চলছে সর্বদায়। দুর্ভিক্ষ সৃষ্টি করে করিয়া কৌশল বৎসরে বৎসরে আসে দারুণ বন্যার জল।। বাড়ি ভাঙে ফসল নেয় বন্যার পানি আসে 化 খাজনা-ট্যাক্সের চাপ দিয়েছে চেয়ারম্যান তহশিলদার লোটাবাটি ক্রোক করে উপায় নাইতো আর।। শোষকের ইমারত গড়তে নেতারা পাগল রঙ-বেরঙেবের হয়েছে ভোটশিকারী দল। কেহ বলে, জাগো বাঙালি উড়াও জয়নিশান বে. ধর্ম গেল জাগো মুসলমান। বাউল আবদুল করিম বলে সূক্ষম রাস্তা ধরো শোষণমুক্ত সমাজ গড়ে বাঁচার উপায় করো।
Farmers, laborers, all brothers of mine, We work hard day and night, yet the food we get does not suffice.

The imperialist, capitalist, and feudalist troops,

They steal the land's wealth together, these three-robber groups.

We fear being jailed or oppressed, if we say things just and fair,

No rice to fill our stomach, no medicines for disease, no clothes to wear.

A rich man's child gets proper education, The child of the poor man dies of starvation.

Clinics full of doctors everywhere, But without money, medicines for the poor are very rare.

Judgements are passed in courts everyday, But judgement for the poor without money? No way.

The ruled poor are left oppressed everyway,

The ruler rules them forever, holding their sway.

Famines they create with some cunning ruse,

Every year terrible floods ferally destroy us.

Houses broken, crops destroyed, the flood water creeps in,

The vicious lenders gloat, knowing that the time to reap their interest now begins. Land and house are sold to the lenders at low rates,

The loan's noose thus binds our poor farmer friends by their necks.

Taxes are imposed and everyday pressed by the administrator,

You have snatched all our belongings, we have nothing more to cater.

To build the houses of exploiters, crazy is the leaders' troupe,

On rounds they have come, the vote hunters' motley group.

Some say "Awake! O' Bengalis, hoist the victory flag!"

"Ruin befalls religion's shrine! Rise Islam!" while others nag.

Some appease the farmer and laborer and workers,

It is all a lie, just vote seeking behaviour. Baul Abdul Karim says, "Choose the finer direction,

Build an oppression-free nation, to live with compassion".

Further, the emergence of pseudo-intellectuals, according to these two songwriters, is harmful to society, as these so-called intellectually enlightened personalities fail to compromise their self-interested activities to act as good examples and to humanely advance 
society. Intellectuals, in the traditional sense, are equated with high moral ground and honesty; Bauls Das and Karim identify such qualities in their idealized moner-manush (man of the heart) - who is simple yet wise, consciously unattached to worldly matters, and committed to social equality. Whereas the pseudo-intellectuals (e.g., educated opportunists) are those who, at the slightest turn of the head, are desperate to forego their principles and ideals to serve their selfish motives. The song speaks on the dubious nature of pseudo-intellectuals heralding treatises of justice and truth, and the equivocal nature of their moral principles where they tend to sway according to the fulfillment of self-interest. Here, Baul Manimohan Das further argues that their ideologies change in the moment that they sell themselves out for material luxuries and newly acquired power, forgetting the needs of fellow humans that are politically powerless.

দেশে বুদ্ধিজীবী কর্তারা সব বিকায়ে গেল,
এদল ওদল করে তারাই বুদ্ধিজীবী হল।।
ন্যায্য কথা বলবো বলে লম্ফঝম্প করে।
সুযোগ পেলেই তখন একটা দলে ঢুকে পড়ে।
তখন মতবাদ সব রাতারাতি বদল হয়ে গেল।
জানি অনেক গুনেগুণী বুদ্ধিজীবী হয়,
আম্মস্বার্থ ভোগবাসনা বুদ্ধিজীবর নয়।
অযোগ্য দান করে জন আর যে করে গ্রহণ,
অনাচারী ব্যভিচারী সমানএই দুজন।
দাশ মণিমোহন খুঁজে বেড়ায় খাঁটি মানুষ কোই
রইল।

দেশে বুদ্ধিজীবী কর্তারা সব বিকায়ে গেল, এদল ওদল করে তারাই বুদ্ধিজীবী হল। সুযোগ পেলেই তখন একটা দলে তুকে পড়ে। তখন মতবাদ সব রাতারাতি বদল হয়ে গেল।। জানি অনেক গুনেগুণী বুদ্ধিজীবী হয়, আঅ্মস্বার্থ ভোগবাসনা বুদ্ধিজীবীর নয়। অযোগ্য দান করে জন আর যে করে গ্রহণ দাশ মণিমোহন খুঁজে বেড়ায় খাঁটি মানুষ কোই রইল।
The intellectual leaders of the country traded their souls, sold themselves out,

They became intelligent anew by changing parties about.

They hark and they roar and they spew, Espouse promises to speak what is true, But with opportune chance they sneak into a group,

And the slew of their ideals and morals held tight, Change its doctrine overnight.

I know that true intellectuals are full of qualities, Contrary to those the pseudo-intellectuals, for whom,

Selfishness, desire, and greed,

Is the ultimate creed.

Those that lend without need,

To the undeserving,

And those that receive,

Are criminals of the same hive.

Both are unjust,

Doers of misdeeds,

And away from morals they tread.

Das Monimohan's tirade is thus,

Looking for a true human being,

Whose intent is genuine.

Through this song Baul Das communicated his commitment to fight for the deprived and downtrodden, as well as questioned the motivations of pseudointellectuals and their hypocrisy. He argued that modern lifestyles often make it difficult for the affluent to participate in welfare activities, for many of them are accustomed to a certain ease of living from which they seldom want to deviate. In this era, in which we observe a wide degradation of morality and ethics, the humane instincts of many (pseudo) intellectuals is often absent. Consequently, such people tend to work with groups that have polar-opposite ideologies, simply because it is conducive to their vested interests.

These two composers further note that the naivety of the poor, who are taken advantage of by others, and wealth depletion and theft furthers the wealth divide, whereby the poor become poorer every day and the wealthy become wealthier. People who are fundamental to building the foundation of a society, the farmers and other unskilled workers, are made to face further difficulties through their material situations, wherein they are burdened with more adversity. These difficulties include a lack of social security in terms of health, finances and assets, as Baul Shah Abdul Karim writes. 


কৃষক মজুর পড়েছে ঘোর আঁধারে
কী করা যায় উপায় বুদ্ধি
মিলেনা আর বিচারে।।
সুদখোর ঘুষখোর মজুতদারে
দালাল টাউট বাটপারে
আগুন দিয়াছে হোদের ঘরে
হয়েছে সাহেব-বাবু
গরিবকে করেছে কাবু
বিনয়ে মানেনা তবু
মরারে আরো মারে।।
দিন হতে দিন আসে কঠিন
এইভাবে আর বাঁচব কয়দিন
আবদুল করিম ভাবতেছে অন্তরে
হয়ে গেলাম নিরুপায়
দুঃখের বোঝা বাড়ছে সদায়
পড়েছি শয়তানি ধোঁকায়
তিন শয়তানের বাজারে।।

The farmers and the laborers have fallen into trouble dark and deep,

What can be done? What is the way? Contemplations or labors of the mind can't reap.

Usurers and grafters,

Pimps and hoarders,

Broker agents and touts,

One and all alike,

Have set fire to our lives,

Without conscience.

Become they now "Master" and "Sir",

The poor they control and haplessly conquer.

Politeness and humility in their hearts have they not one bit,

Beating the nearly dead into death, is far worse than unfit.

Morning goes as it comes along,

Such hardships the life wouldn't endure for long,

Abdul Karim thinks within,

“Helpless, we are now,

The burden of sorrow up and up evergoes,

We have just walked into three devils' hoaxes.

He wrote the song by depicting peoples' sufferings in and around his region, and it is rooted in his own experience and reflection. In remote villages, exploitation still exists, in their primitive and contemporary manifestations, including through political intimidation, threats, and bribery, along with other forms. Oftentimes, exploitation in modern societies is less tangible, where financial traps are placed and promises are broken unethically by the dominant stakeholders, and many of the poor have been robbed of what few possessions they had. Moreover, the lack of adequate education about policies and governance and an uncritical trust in the power structure aggravates their situated adversities. Such a scenario disadvantages common people as they fail to identify ways to overcome their hardships.

The last song for this theme was written by Baul Manimohan Das, in which the poet ironically speaks of the mentality of tyrants and the powerful who exploit poor people. With total antipathy to the impoverished, the moneylenders and other such similar rapacious people flourish at others' misery and present no shame or moral reservations. The song suggests that the unscrupulous rich exploit their positions of power and deploy methods of duplicity and deceit to entrap the unwitting poor.

Since his childhood, Baul Das experienced financial hardships and observed how financially powerful people led to ruin in the lives of economically disadvantaged populations. Depicting the unfortunate and turmoiled realities of his contemporary period, as well as the abuse of power and authority, and of extortionist mindsets, the Baul poet plays the devil's advocate to evoke the truly ethical and reflexive behaviors. Tyranny relies on its own power to silence the victim, and the song can be seen as an example where subjugations can be muzzled through speaking out against such misdeeds. The next theme emphasizes the aspect of communicative engagements, particularly in situations where the voices of the marginalized are delegitimized, if not erased, in various spaces of discursivity. 


ও গিন্নি সারা দেশ ডুবে গেছে তাতে কী
হয়েছে,
গিন্নি আমার তো লাভ আছে।
পাড়ার লোকে ধার নিতে সব আসবে আমার
কাছে।
যত লোকের হয় সর্বনাশ আমার তৈ্যষ্ঠ মাস,
লোকের যদি হয় গো ভালো আমার সর্বনাশ।
লোকে গালমন্দ যতই দেয়না তাতে রাগের কী
আছে।।
লোকে শুনি আমায় বগে পয়সার পিশাচ,
আমার তাতে হয় নাগো রাগ বরং আগে হাস।
জগতের লজ্জা ঘৃণা নাইগো যাদের তারাই
বড় হয়েছে।।
দেখে শুনে বলে ওগো দাস মণিমোহন,
নিকৃষ্ট মানুষ যারা তারা করেগো শোষণ।
শোনো শোষকেরা এমনি করেই শোষণ করে
চলেছে।।

Oh! dear wife, the whole country in misery has drowned.

But, so what, oh darling, in it profit I have found. Now everyone will come to borrow money, And in debt to me they will be bound.

The more people are in distress and they perish, The more will I gain, the more I flourish, If good befalls them and they do better, Then my good times will come to a finish. Berate me they can as much as they do, Such things give no cause to rile my sinews. Often, I hear people say that I am a bloodsucker for money,

Angry? Never, but to me that is funny. Those that have no shame or pity in their stride, In this world they become something with much pride.

Seeing and hearing all of this, says Das Monimohan, Lowly beings are those who indulge in exploitation. Listen, this is how the exploiters are continuing their oppression.

\subsection{Communicative Aspects}

Bauls Das and Karim express alarm over the never-ending sufferings of the underprivileged, as a result of societal power dynamics. They notice that the rich and the corrupt do not hesitate to destroy and exploit the marginalized population to realize their capitalistic agendas and maintain the status quo. Their voices and performances can be seen as a catalyst for raising conscience at the margins. They embrace communicative avenues and foreground anti-hegemonic poetics, raising anti-dogmatic narratives. We have seen the resonance of such ethos in the words of Shah Abdul Karim, where he declares, "I am raising my voice to fight against the power structure that creates and reinforces marginalization. Previously, I was entirely invested in worshiping tattwas; but over the years, I developed a conviction that I have to work for the deprived and penniless. Together, we have to resist the oppressors, greedy, and ill motivated powers. I always want to resist the abusers and the tyrants, and sing for the oppressed; that is how I want to travel in my uncompromising rebellious journey". While raising their voices against societal injustices they emphasized self-awareness and mindfulness. Reflecting on his own journey, Baul Manimohan Das said, "I reminded my fellow Bauls to live an ethical and honest life by practicing what they preach in their own lives. Personally, I believe in living a simple life. I never spend money to buy expensive clothes or food, and never indulge myself in any addiction".

The de-humanization of the poor occurs at the expense of a person's morals, and the poor suffer and continue to be exploited. The marginalized, deceived easily by the powerful, ultimately remain deprived of basic privileges as they are denied the outcomes that have been promised. In his personal life, Baul Shah Abdul Karim experienced several instances of rejection and humiliation, evidenced in his writing. Through this song, he pays attention to discursive violence and seeks to raise his voice against such injustice and exploitation. 


\author{
গরিবের কী মান-অপমান দুনিয়ায়? \\ গরিবের নাই স্বাধীনতা পরাধীন সে সর্বদায়।। \\ ভোট নেওয়ার সময় আসিলে নেতা সাহেব তখন \\ বলে \\ এবার আমি পাস করিলে কাজ করব গরিবের দায় \\ পরে লাইসেন্স পারমিট দেওয়া ধনীর বাড়ি খাসি \\ খাওয়া--- \\ সালাম দেওয়া নৌকা বাওয়া এইমাত্র গরিবে পায়।। \\ অস্থিচর্ম সার হয়েছে রক্তমাংস চলে গেছে \\ প্রাণটি শুধু বাকি আছে কখন জানি চলে যায় \\ আবদুল করিম ভাবছে মনে কার দুঃখ কেবা শোনে \\ স্বার্থের ব্যাপার যেখানে দয়ামায়া নেই সেথায়।।
}

\author{
What respect or disrespect is \\ For the poor who in this world inhabit. \\ The poor can't partake in freedom universal, \\ Being shackled to oppression eternal. \\ When the time to receive votes comes near, \\ Says our political leader so dear- \\ 'if we do win this time, \\ Work we would with all our might, \\ To make the future of the poor people bright.' \\ And after that when the time comes, \\ The rich get the lion's share. \\ Permits and licenses, all they get, \\ The poor, they completely forget. \\ Goat meat and other things, on them they \\ feast, \\ The poor only get to offer salutations, row \\ boats, \\ Trampled under their feet, respected the least. \\ Flesh and blood have disappeared, the body \\ has come to skin and bones, \\ Life's flame is all that barely there is, \\ Who knows, at any moment, even that might \\ extinguish. \\ Abdul Karim thinks to himself, "Whose grief \\ does anyone hear, \\ Where self-interest lives, humanity withers".
}

The song argues that electoral fraud and manipulation, devised by dominant stakeholders, impoverishes the marginalized even further. The political 'benefactors' use the poor to ensure their success and to their benefit, and, after securing political success, the common people are systematically ignored and forgotten, which leads to hopelessness and dissatisfaction for the marginalized. The poet, by discussing the lack of freedom and the compromised dignity of the underprivileged, searches for an empathetic confidant who acknowledges the agony and distress at the margins.

Throughout his life, Baul Shah Abdul Karim witnessed wars, famines, and moments of large-scale socio-economic crisis. His songs cannot be seen only as testimony of such incidents, as they are also reflective of deprive peoples' helplessness and misery. This composition of Baul Karim, a diatribe against the injustice meted out to the impoverished, indicates the generic degradation of society through oppression and tyranny. It illustrates the way society works, in the name of democracy, whereby the rich, who enjoy positions of power, display an apathy for the poor. Without freedom, the poor are cheated and exploited, and thus find it difficult to have faith in anyone.

The song, through a critical lens, points out that in a capitalistic system, it is difficult to judge a friend from an enemy. An enemy often appears friendly for the purpose of exploitation. Those that speak the truth are often ostracized and intimidated by the powerful. Baul Karim succinctly criticizes the unethical behavior of the financially powerful stakeholders, who, while fulfilling their vested interests, strategically ignore and systematically ruin the lives of the marginalized.

The next song speaks to a lack of freedom as the villagers become destitute and deprived. Indicating the scenario of internal colonization, the poet opines that common people become voiceless and slaves in their own land. In such a situation, the lack of support from fellow humans eventually becomes a source of tremendous angst and agony. Baul Shah Abdul Karim argues that power resides with those who have resources; particularly, monetary aspects are valued more highly than moral or social qualities. 


\begin{tabular}{ll}
\hline কে বা শত্রু কে বা মিত্র & Who is a foe and who is a friend? \\
বুকে উঠা দায় & Such a quandary it is to understand. \\
তাইতো দেশের অবনতি & Hence the state of this country, \\
সাধুর নিশান চোরের নায়। I & Where thieves disguise as sage. \\
স্বার্থপর শত্রু দলে & The selfish villains, \\
দেশে দিছে আগুন জেলে & Have set the nation on fire. \\
উচিত কথা বলতে গেলে & If one says things are just and fair, \\
তারা আবার চোখ রাঙায়।। & They are always met with a frown or a glare. \\
কেউ হইল কালোবাজারি & Some are black marketers and some do hoarding, \\
কেউ করতেছে মজুতদারি & Some steal money from relief funds, too, without \\
কেউ করতেছে রিলিফ চুরি & missing a chance. \\
যে যেভাবে সুযোগ পায়।। & If I might hope for some censure, \\
শান্তি পেতে আশা করি & In reality I am in even more danger. \\
আসলে বিপাকে পড়ি & Of self-interests they are peddlers, \\
স্বার্থ নিয়ে মারামারি & Nothing comes to naught. \\
শেষ হয়না তাদের বেলায়।। & The poor men's questions do not arise, \\
গরিবের প্রশ্নই নাই & Whether they live or die from starvation or disease. \\
বাঁচি কি-বা মরিয়া যাই & Abdul Karim says, “Oh, brother, \\
আবদুল করিম বলে রে ভাই & In the village of old, \\
সোনা বর্ষে সোনার গায়।। & We will always have fields of gold”. \\
\hline
\end{tabular}

Experiencing the inequality within the modern capitalistic society, the those who are helpless are impoverished and trapped in an endless struggle for human rights and freedom, left to seek a solution to their predicament, in which all resources and necessities are scarce. The poignant situation of poverty as its own form of tyranny binds the impoverished in an eternal struggle for freedom from dominance. While negotiating with unending pain and suffering, the Bauls uncompromisingly raise their voices towards ensuring the human rights, material and spiritual sustenance, and survival of the underserved. The final theme focuses on the emancipatory pronouncements of Bauls Das and Karim in realizing their vision of a free and liberated future.

\begin{tabular}{|c|c|}
\hline আমার দেশে কেন আমি & Why, in my own land, \\
\hline কাঙাল হলে মরে & Have I turned a beggar? Alas, I could not withstand! \\
\hline নাই কেন মোর সহায় সম্বল & Why do I have no shelter, no help? \\
\hline সদায় ভাবি রে।। & I keep thinking thus. \\
\hline নাই কেন মোর স্বাধীনতা & Why do I have no freedom? \\
\hline বুকে নিদারুণ ব্যথা & My heart aches so. \\
\hline কার কাছেকই দুঃখের কথা & Who do I tell my stories of pain? \\
\hline কে শুনিবেরে।। & Who would listen to that in vain? \\
\hline এই দেশে জন্ম আমার & I was born in this land. \\
\hline আমার কেন নাই অধিকার & Yet why do I have no right? \\
\hline জীবন নিয়ে বেঁচে থাকার & To survive by day and night, \\
\hline উপায় নাই রে।। & There is no way. \\
\hline দুঃখে গড়া জীবন নিয়া & Life is steeped in harsh pain, \\
\hline চিরদুঃখের অধীন হইয়া & Under such eternal distress, \\
\hline দুঃখের বারোমাসি গাইয়া & With songs of sorrow and sadness. \\
\hline জীবন গেল রে।। & Years of torment pass again and again, \\
\hline গরিবের দুঃখ যত & The poor with their suffering and pain, \\
\hline কার কাছে বলিবে কত & To how many people can you explain? \\
\hline করিম বলে আমিও তো & Karim says, \\
\hline মানুষ ছিলাম রে৫@ & "A human being I once was, too" \\
\hline
\end{tabular}

\subsection{Emancipatory Visions and Calls}

As persistent marginalization causes tremendous anxiety and stress, Bauls Das and Karim have embraced discursive methods to communicate with the common people to raise awareness and consciousness. On one hand, they disrupt the hegemonic portrayal of the marginalized as inferior and devoid of agency; and, on the other hand, through 
raising consciousness, Bauls Das and Karim create avenues for change and emancipation both materially and discursively. Baul Shah Abdul Karim reiterated that a "Baul's fundamental duty is to raise consciousness among commoners". He added, "whether they (the discourses) work or not, I believe it is my duty to raise a voice". Even endless suffering and stress could not stop them from dreaming or communicating their dreams. For instance, Baul Das once said, "I dream of a future where the downtrodden people, specifically the underprivileged children, can live without hunger, malnutrition, and domestic violence". On a similar note, Baul Karim's fearless voice inspiringly pronounced, "I am hopeful; I am sure that human liberation will be realized one day. Monstrous devils will be defeated. The good time will come someday. I dream for a society that is harmonious, peaceful, and liberated". The next few songs are examples of such pronouncements and convictions.

In a pastoral tone, the following song converses with village populations, whose livelihood and survival are dependent on their meaningful utilization of natural resources. With a tone of positivity, hope, and optimism, the song advocates hard work and united efforts as a possible means to bring about transformation at the margins. Thus, in a warm, energetic, and enthusiastic way, Baul Karim sought to offer a new ray of hope in the minds of the underprivileged.

ওরে চাষি ভাই, শক্ত হাতে লাঙ্গল ধরা চাই
যত্ন বলে রত্ন ফলে পরিশ্রনে প্রাণ বাঁচাই ।।
উৎপাদনের প্রয়োজনে চলো এবার সর্বজনে
মাটির সনে মনেপ্রাণে আমরা করি লড়াই ।।
কৃষক মজুর সবাই মিলে আছি বাংলামায়ের
কোলে
পরিশ্রমে সোনা ফলে তবে কেন দুঃখ পাই । ।
মাছ ফলাও গাছ লাগাও যত পারো সবজি
ফলাও
পাট ফলাও তুলা ফলাও ধান সরিষা বুট
কালাই ।।
কাজ করে যাও মনোবলে
কৃষক-মজুর-তাঁতি-জেলে
বাউল আবদুল করিম বলে এ ছাড়া আর
উপায় নাই।।

Oh! my farmer brother, plough the grounds with hands stern,

With able care and immense passion, treasures we will unearth,

Saving lives, giving life, is our primary conduct. The call for produce is upon us, let us brothers join hands,

Let us wrestle with the earth, to beget Nature's bounty grand.

Farmers and laborers us all,

Cradled in our Mother Bengal's loving bosom, Through hard work and perseverance, we can cultivate gold,

Alas, but even then, we are stricken by sadness bold. Farm fish, plant trees, grow vegetables,

Reap jute, pluck cotton,

Grow wheat and grow mustard.

Farmers and laborers,

Fisher-men and handloom weavers,

Work to your fullest,

Put all your emotional strength,

Baul Abdul Karim says,

Sans this there is no other way.

Through his song, Baul Abdul Karim argues in favor of reducing dependence on others, inculcating self-dependence as a potential route for survival. Paying attention to agriculture and village industries, he calls for a sustainable and strengthened rural economy and an enhanced quality of life.

Baul Shah Abdul Karim has further noted that the marginalized lack access to basic amenities, such as food, shelter, water, and even civil rights and justice. Baul Karim points out the futility of seeking truth in an autocratic environment, where the powerful manipulate the system for motivated by selfish interests. According to him, the threat of state oppression is an added factor to the reality of starvation and mass hunger in the villages. In such a state, however, the song argues the machinery of authority and power exert greater strain on the poor, rather than providing meaningful support for them. 


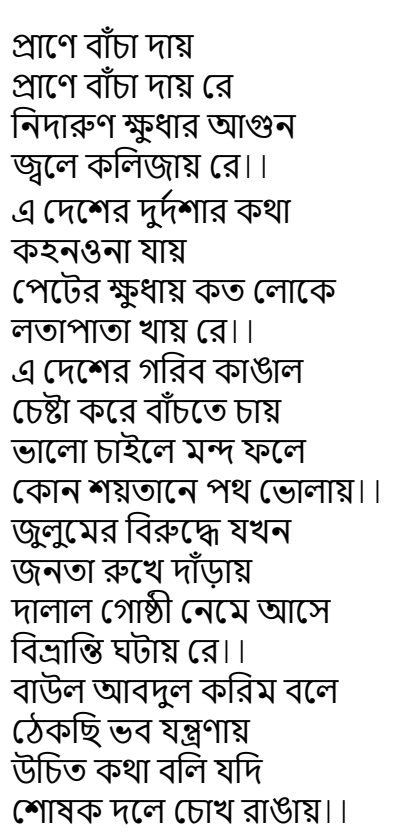

It is hard to survive,

It is hard to survive, oh!

Insatiable hunger pains,

In our hearts they burn bright.

Unspeakable is the adversity,

Upon this nation bestowed,

To eat grass, twigs, weeds, and creepers-

Hunger makes many people to resort.

The poor and the needy of this country,

Try by effort to thrive.

A good deed wished,

Often bears rotten fruit,

As a charming devil,

Plays on an ulterior flute.

When against oppression,

The people stand upright,

The agents in power attack,

Bringing chaos in their stride.

Baul Abdul Karim says,

"Stuck we are in an eternal impasse,

If we speak what is true and what is right,

The oppressors inflame us with their might".

To Bauls Das and Karim, this is a continuous reality for those surviving on the margins of the society. Malnutrition and a lack of health facilities often act as the reason for a high mortality rate within lower socio-economic groups. Since economic development for them has stagnated, inflation perpetuates and amplifies poverty. Baul Abdul Karim argues in favor of individual as well as societal forms of resistance, potentially challenging the status quo, and to bring about meaningful changes in the lives of the downtrodden.

In his own life, Baul Karim refused to succumb to endless suffering and adversity; his voice emerged as a fearless pronouncement of creating a hopeful future. This final song depicts the impoverished realities of farmers that toil on the land all day long. It also portrays how the constant pain and the struggle for life, the economic constraints, and its consequences manifest at the margins. Critiquing the commercialistic practices of the mainstream, Baul Abdul Karim calls for a better civilization, one that is free of exploitation.

The song points at political injustice, the ever-increasing gap in wealth distribution, and moral deterioration. To ensure survival and sustenance in underserved spaces, he asserts his firm belief of love and peace as tools to pave the way for an equal and harmonious society.

With their simple tunes and accessible language, unlike many esoteric songs, songs such as those quoted above, the Bauls appeal and to inspire common peoples, to overcome the inner compulsion of greed and the markers of desires, thereby creating a habitable and just world. The liberatory humane utterances of these songs are meant not only intended for spiritual development but are also instrumental for imagining and realizing structural transformations and subaltern emancipation. 


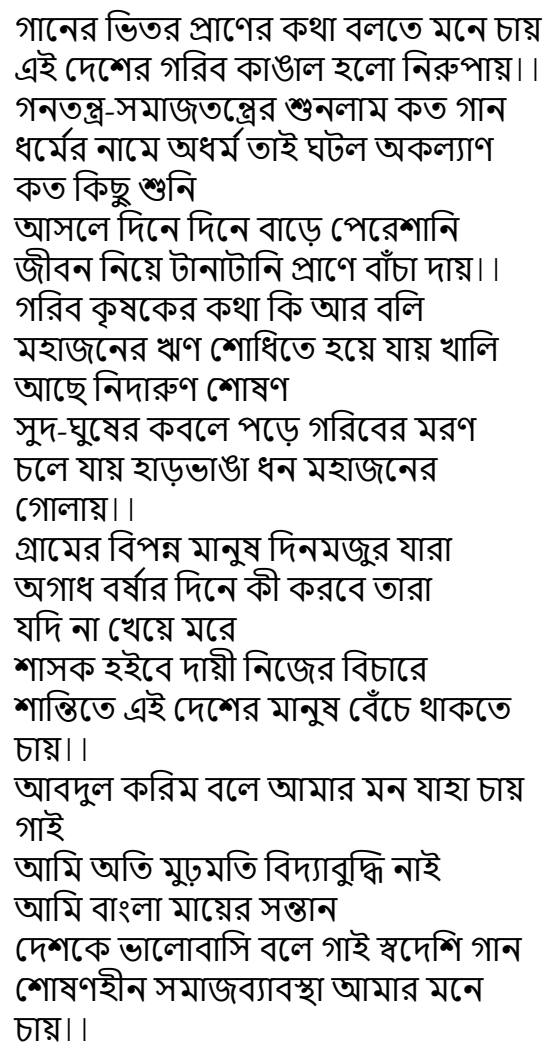

The heart wants to speak out loud through a musical strain,

The wretched of this nation have become helpless and vain.

Of socialism and democracy, I have heard many tales, Injustice in justice's name, thus bring disgrace.

With every passing day, I hear so many things,

Day by day hardships increase and gain more powerful wings.

Tug of war with life we play,

Sustenance becomes difficult day by day.

Oh, what do I tell of the sad farmer,

Penury befalls him,

in repaying all debts to the money-lender, savings become slim.

There is deep exploitation, to high interest and bribes they are prey,

So the poor keep dying in this nation,

Their fates have gone astray,

Their hard-earned wealth goes to the hoarder's den. Endangered people of the village,

who are daily laborers, workers, masons, working for a daily wage.

What would they do during endless heavy showers? If they starve to death, losing life's powers.

The ruler only by himself would be responsible,

Peace of mind is what is sought by people.

Abdul Karim says "In my song my heart's passions are caught,

Neither too educated nor too bright I am not, A son of my Mother Bengal, I love her much,

Hence sing patriotic songs as such.

I dream and long,

For a society free of oppression,

For a society free of tyranny and exploitation".

\section{Discussion}

Historically, the feudalistic and colonial power structures attempted to banish Bauls, both physically and symbolically, from society. In response, the Bauls, and similar communities of Nera-Neri, Sahebdhani, and Kartabhaja, use their songs and discourses as weapons through which to resist such oppressions and exploitation (Jha 2014). By no means are such communicative acts homogeneous and formulaic in nature, but, instead, they are creative and emerge so as to meaningfully combat contextual challenges. In their consistent struggle against challenges and adversities, their musical performances and communicative practices prove foundational to foregrounding their emancipatory worldviews, rationalities, and imaginings, as well as their ideological rebellion and nonconformist commitments (Dutta and Dutta 2019). By negotiating with a variety of socioeconomic and/or politico-religious threats, Baul songs, particularly those of a non-esoteric composition, from the margins of the global South, often legitimize their anti-caste and pro-human articulations through their songs.

Borrowing from Scott (1990)'s theories, we might infer that the poetic communications and aesthetic performances of the Bauls, especially those who write the kind of songs discussed here, can be seen as "stubborn bedrock" of skepticism and dissent, as well as the key agentic mechanism for imagining sustainable transformation. The musical performances and the discourses of such Bauls, therefore, emerge as potential sites of contestation and critique. Here, the economically disadvantaged and oppressed population find avenues to raise their voice against orthodoxies of dominant religions, more specifically Hinduism 
and Islam, as well as the age-old caste system and religio-politically motivated communal violence. Being on the fringes of the dominant society, these Bauls did not limit themselves to the religious realm. Rather, they expressed and express their rebellion against conventional socio-political norms, power dynamics, and the status quo. As social outcasts, beggars, and wanderers, they, by dismantling and rupturing religious doctrines and boundaries and by challenging the dominant mainstream discourses, established themselves as iconoclasts (Urban 2001).

We can observe that the concerns of Bauls Das and Karim are echoed in many global indices. For instance, the HDI (Human Development Index), which reflects the nationstate's average achievement in three key dimensions - namely, health, education, and standard of living, ranks India and Bangladesh as 131 and 133, respectively (among 189 countries) (UNDP 2019). Similarly, as per the Global Hunger Index, Bangladesh and India rank 75 and 94, respectively (out of 107 countries), which is a matter of serious concern. Moreover, in the context of corruption, the Transparency Index demonstrated that Bangladesh's rank is 146 (among 180 nations), while the realities of the rest of the Bengal are not very different. While all the indices indicate the overall scenario for both urban and rural spaces combined, the situation of rural Bengal is, understandably, worse than the overall (i.e., urban + rural) situation.

Over the centuries, the Brahmanical, colonial, and neoliberal forces consistently used age-old as well as innovative tricks to divide and rule subaltern populations. Deliberate exploitative discourses and unapologetic immoral actions characterize the cardinal makeup of the oppressors, who orchestrate structural and strategic oppressions and abuse both overtly and covertly, where the poor, the victims, are only pushed further to the margins (Mukharji 2012). Through their songs, Bauls Das and Karim foregrounded the hopelessness and helplessness at the margins; particularly in instances where the voices and agencies of the socio-economically weaker populations are systematically delegitimized, and in cases where the poor do not have a meaningful lobby or the financial strength to resist dominant powers. In the contemporary era, ethical bankruptcy emerges as a key concern as so-called educated and intellectual communities exhibit no or little moral adherence to ideological doctrines. Capitalistic and neoliberal values and markers of desires compel many such opportunists to espouse the equivocal nature of moralistic commitment and thereby conveniently fulfill their vested interests. Consequently, the working-class populations that constitute the foundation of our society are subject to poverty, and are also increasingly burdened with pain, abuse, and grief.

While the dehumanized populations are trapped within the confines of poverty, the absence of humanity worries both Bauls Das and Karim, as they decry that in such inescapable misery there is no empathetic ear to listen. They criticize, through music, the unwise and unjustified tendencies of a society that values wealth and exuberance over ethical, moralistic, and intangibly valuable facets of our existence. In this era of increasing corruption, class enmity, cheating, and exploitation, they place an emphasis on communicative aspects to create social awareness. In this world, where we are presented with hyperbole and excess, they stress how the unfulfilled promises from the powerful and the strategic ignorance from the mainstream leaves the poor uncared for, unattended to, and forgotten. Using plain rustic language and melodies, they not only seek to rupture the constant fear that the underserved experience in their everyday existence, but also to dismantle the barriers presented for speaking the truth out loud.

As suicide and agitation in farmer and the laborer communities has become commonplace in contemporary South Asia in the last few decades, Bauls Das and Karim point to the necessity of building a network of solidarity and united efforts to fight for a society that is free from all forms of exploitations and injustices. In recent times, in which the activists who foreground the issues of the underserved have either been silenced or made to disappear, both Bauls Das and Karim have chosen an aesthetic route to articulate the truth of oppression through cultural means. They discursively legitimize the futility of speaking the truth in an unjust and unequal society, where the powerful continually use methods of 
manipulation to satisfy their desire for dominance. Communicatively, they have depicted the reality of the poor being muted, and thereby have argued in favor of bringing forth voices, opinions, values, as well as agencies to the spaces of discursivity. Highlighting our society's failure to devise a sustainable and exploitation-free world, both of them, through their songs, communicate their visions of achieving a society cured of oppressions, abuses, and corruption. Upholding the unending and untiring efforts of the marginalized populations to secure basic survival requirements, they, through their poetic imaginings, dreamt of a self-reliant future.

Such emancipatory pronouncements and performances not only aim to inspire the masses to organize for freedom, but they also aim to guide the common people to take ownership in raising their voices to improve their communities. In their own lives, both of these Bauls have tried to organize people and fellow practitioners to improve and a positively impact the lives of the oppressed. In the words of Baul Shah Abdul Karim, "I performed to create awareness and consciousness. I performed to give voice to the voiceless, I performed to remember those who have sacrificed their lives during the struggle for freedom. That is how I supported the Muktijuddho (freedom struggle of Bangladesh) and encouraged the fighters". They, along with other senior Bauls, through their songs and discourses, inspired and motivated Bauls and other performers to organize against injustices to claim their human rights during the last few decades. Consequently, many Baul organizations were formed across Bengal, and they collectively participated in social movements to promote their voices. For instance, Jha (2014) noted that in 1986, many Bauls marched on foot from Katra village of Murshidabad to Kolkata, the capital of West Bengal, to create awareness about their ideology and performances. While this paper is concerned with literary analysis and archival research, future scholarship might engage more ethnographically and examine the transformative impact of Bauls Das and Karim's and their discourses in people's lives.

By rejecting the tendency of some scholars to depict Bauls as "asocial, ahistorical... esoteric sects" (Mukharji 2012, p. 47), we might argue that their call for freedom, joy, universal brotherhood, mutual respect, and love may potentially transcend existing socioreligious and political boundaries, spiritually, culturally, and psychologically. Bauls, the so-called 'deviant' and playfully subversive minstrels, in their path of unorthodox spirituality, consistently engage with unsettling and uncomfortable questions. Questioning the mainstream's intentions and acts to portray underrepresented spiritual discourses as lowly and filthy (specifically for their sexo-yogic practices), both the Bauls ceaselessly challenge and interrogate the material and communicative absences and disparities at the margins. By engaging communicatively with impoverished and oppressed populations, they have actively tried to reclaim the voices and agencies of the subaltern population's possibilities for meaningful transformation. Life experiences and nuanced understandings of situated adversities and abuses guide both the singers to connect with the local (e.g., the rural and underserved communities in their vicinity), as well as a wider audience, regionally, nationally, and internationally. These mindful iconoclast's resilience, astute consciousness, and their uncompromised faith in liberation and emancipation guide them to musically imagine a society that is free from the barriers of caste, religion, hierarchy, as well as all forms of dominations and discrimination.

Author Contributions: Conceptualization and methodology, U.D.; writing-original draft preparation, review and editing, U.D., S.G., P.G., P.B., S.S. and S.M. All authors have read and agreed to the published version of the manuscript.

Funding: This research received no external funding.

Acknowledgments: The authors are immensely grateful to Terry Christenson and Ashabari Ray for their invaluable suggestions and inputs that greatly improved the manuscript.

Conflicts of Interest: The authors declare no conflict of interest. 


\section{Notes}

1 Several tattwas [tattwa- theory/epistemology of ] are represented and discussed in Baul songs. They talk about-"deha-tattwa" [body], "guru-tattwa" [spiritual teacher], "gujhyo-tattwa" [esoteric], "prema-tattwa" [spiritual love], "srishti-tattwa" [creation], and "param-tattwa" [supreme/almighty] (Sengupta 2015).

2 Twilight language: the term portrays the essence of Bauls' mindful/strategic usages of language, which they communicate in an ambiguous/hidden/esoteric and/or (intentionally) reverse/veiled way (Dutta 2019).

\section{References}

Akter, Md. Shaon, Md. Asgor Hussain, and Md Sanaullah. 2017. Cleverness of Lalon. Imperial Journal of Interdisciplinary Research 3: 580-85.

Capwell, Charles. 1974. The esoteric beliefs of the Bauls of Bengal. The Journal of Asian Studies 33: 255-64. [CrossRef]

Capwell, Charles. 1988. The popular expression of religious syncretism: The Bauls of Bengal as Apostles of Brotherhood. Popular Music 7: 123-32. [CrossRef]

Chakraborty, Dipesh. 2000. Provincializing Europe: Postcolonial thought and Difference. Princeton: Princeton University Press.

Charmaz, Kathy. 2000. Grounded theory: Objectivist and constructivist methods. In Handbook of Qualitative Research. Edited by Norman K. Denzin and Yvonna S. Lincoln. Thousand Oaks: Sage, pp. 509-36.

Das, Manimohan. 2014. Gramin Sangiter Dali (Bengali). Kolkata: Lok Sanskriti O Adivasi Sanskriti Kendra.

Dutta, Uttaran. 2019. Sufi and Bhakti performers and followers at the margins of the Global South: Communication strategies to negotiate situated adversities. Religions 10: 206. [CrossRef]

Dutta, Uttaran, and Mohan Jyoti Dutta. 2019. Songs of the Bauls: Voices from the margins as transformative infrastructures. Religions 10: 335. [CrossRef]

Freire, Paulo. 1970. Pedagogy of the Oppressed. London: Routledge.

Fulton, John. 1987. Religion and politics in Gramsci. Sociological Analysis 48: 197-216.

Grover, Mansi. 2015. An analysis of transgressive voice in Sufi poetry. International Journal of Interdisciplinary and Multidisciplinary Studies 2: 85-87.

Hossain, Amzad, and Dora Marinova. 2012. Grassroots cultural policy for water management in Bangladesh. Water Practice and Technology 7. [CrossRef]

Hossain, Md Amzad, and Dora Marinova. 2005. Poverty Alleviation-A Push Towards Unsustainability in Bangladesh? In Proceedings of International Conference on Engaging Communities. Brisbane: Queensland Department of Main Roads.

Imam, Shubhendu. 2013. Shah Abdul Karim Rachanasamagra (Bengali). Sylhet: Boipatra.

Jha, Shaktinath. 2014. Baul-Fakir Dhwongso-Andoloner Itibritto (Bengali). Kolkata: Manfakira.

Khalid, Md Saifuddin, and Md Saiful Alam Chowdhury. 2018. Representation of intangible cultural heritage of Bangladesh through social media. Anatolia 2018: 1-10. [CrossRef]

Kumar, Mukesh. 2019. The Art of Resistance: The Bards and Minstrels' Response to Anti-Syncretism/Anti-liminality in north India. Journal of the Royal Asiatic Society 29: 219-47. [CrossRef]

Larson, Gerald. 1995. India's Agony over Religion. Albany: State University of New York Press.

Lincoln, Yvonna S., and Egon G. Guba. 1985. Naturalistic Inquiry. Newbury Park: Sage.

Lorea, Carola Erika. 2013. "Playing the Football of Love on the Field of the Body": The contemporary repertoire of Baul Songs. Religion and the Arts 17: 416-51. [CrossRef]

Lorea, Carola Erika. 2014. Searching for the divine, handling mobile phones: Contemporary lyrics of Baul songs and their osmotic response to globalisation. History and Sociology of South Asia 8: 59-88. [CrossRef]

Lorea, Carola Erika. 2015. If People Get to Know Me, I'll Become Cow-dung: Bhaba Pagla and the songs of the Bauls of Bengal. Puspikā: Tracing Ancient India through Texts and Traditions: Contributions to Current Research in Indology 3: 119-42.

Lorea, Carola Erika. 2017. The difference between a pandit and a wise man: A study of Bengali songs as literature against literature. Cultural Studies 1: 1-15.

Mahbub-ul-Alam, Ahmad, Mohammad Ehsanul Islam Khan, and Md Tariqul Islam. 2014. A study on spiritualism in the lyrics of Shah Abdul Karim. International Journal of Multidisciplinary Research and Development 1: 29-33.

Majumdar, Dipak, and Gaston Roberge. 1979. The Bauls: Communicators and Artists. National Journal for the Performing Arts of Bombay 1979: 1-14.

Manuel, Peter. 2008. North Indian Sufi popular music in the age of Hindu and Muslim fundamentalism. Ethnomusicology 52: 378-400.

Mondal, Anirban. 2013. Lalon, Lalon geete, and society: Ahumanitarian socio-philosophical discourse. The Criterion 12: 1-7.

Mukharji, Manjita. 2012. Ek rajye hale dujona raja, kar hukume gata hoy praja: Metaphors of everyday peasant resistance in Baul songs of colonial Bengal. South Asian History and Culture 3: 47-69. [CrossRef]

Novetzke, Christian Lee, and Laurie Patton. 2008. Subaltern. In Studying Hinduism: Key Concepts and Methods. Edited by Sushil Mittal and Gene Thursby. Abingdon-on-Thames: Routledge, pp. 378-99.

Openshaw, Jeanne. 2002. Seeking Bauls of Bengal. Cambridge: Cambridge University Press.

Patnaik, Arun. 2014. Tagore, Folk-religion and subalterns: Potentialities for Indian secularism. Journal of Polity and Society 6: 20-28. 
Salomon, Carol. 1995. Baul songs. In Religions of India in Practice. Edited by Donald S. Lopez Jr. Princeton: Princeton University Press, pp. 187-208.

Scott, James C. 1990. Weapon of the Weak Everyday Forms of Peasant Resistance. New Haven: Yale University Press.

Sengupta, Jayita. 2015. The Baul sursadhak: The tradition and individual talent. Journal of Literature and Art Studies 5: 151-57.

Shaker, Himadri. 2020. Baul Ranesh Thakur's music room torched in Sunamganj. Dhaka Tribune. May 19. Available online: https:// www.dhakatribune.com/bangladesh/nation/2020/05/19/Baul-ranesh-thakur-s-music-room-torched-in-sunamganj (accessed on 15 November 2021).

UNDP. 2019. Human Development Report 2019. New York: UNDP.

Urban, Hugh B. 2001. The marketplace and the temple: Economic metaphors and religious meanings in the Folk songs of colonial Bengal. The Journal of Asian Studies 60: 1085-114. [CrossRef]

Wang, Georgette. 2010. De-Westernizing Communication Research: Altering Questions and Changing Frameworks. London: Routledge.

World Bank. 2017. West Bengal Social Inclusion. Available online: http://documents.worldbank.org/curated/en/382741504252542549 /pdf/119345-BRI-P157572-West-Bengal-Social.pdf (accessed on 15 November 2021). 